\title{
Componentes do apoio matricial e cuidados colaborativos em saúde mental: uma revisão narrativa
}

\author{
Components of matrix support and collaborative mental health \\ care: a narrative review
}

Sonia Augusta Leitão Saraiva (https://orcid.org/0000-0002-2305-9246) ${ }^{1}$

Jorge Zepeda (https://orcid.org/0000-0002-3279-0372) ${ }^{1}$

Alberto Fernández Liria (https://orcid.org/0000-0003-2978-3777) ${ }^{2}$

${ }^{1}$ Leeds Institute of Health and Sciences (LIHS).

University of Leeds, Level

10, Worsley Building,

Claredon Way. LS2 9NL

Leeds Reino Unido.

soniasaraiva@hotmail.com

${ }^{2}$ Universidad de Alcalá.

Madrid Espanha.

\begin{abstract}
Collaborative care between mental health and primary care is effective in improving health outcomes. The matrix support has similarities with collaborative care little explored in the literature. This article compares the two models and analyzes the matrix support from evidence on collaborative care. Narrative review. Components of each model (activities and dimensions) were identified and compared. Evidence on collaborative care informed analysis of similar components of matrix support. The dimensions of the matrix support - educational support, specialized care, regulation, co-management - and collaborative care - multiprofessional care, systematic communication, structured care, organizational support - were identified. The main similarity between the models lies in the direct collaborative activities around clinical problems which is related to effectiveness in collaborative care studies. Direct collaborative activities are a positive aspect of matrix support. Structured care and support at the organizational level should be encouraged. Future studies should refine the proposed categories and explore their use for the development of matrix support.
\end{abstract}

Key words Primary health care; Mental health services; Review; Collaborative care; Matrix support
Resumo Cuidados colaborativos entre saúde mental e atenção primária são efetivos em melhorar desfechos de saúde. O apoio matricial tem semelhanças com cuidados colaborativos pouco exploradas na literatura. Este artigo compara os dois modelos e analisa o apoio matricial a partir de evidências sobre cuidados colaborativos. Revisão narrativa. Componentes de cada modelo (atividades e dimensões) foram identificados e comparados. Evidencias sobre cuidados colaborativos informaram análise de componentes semelhantes do apoio matricial. Foram identificadas dimensões do apoio matricial - suporte educacional, cuidado especializado, regulação, cogestão - e dos cuidados colaborativos - cuidado multiprofissional, comunicação sistemática, cuidado estruturado, suporte organizacional. A principal semelhança entre os modelos está nas atividades colaborativas diretas em torno de problemas clínicos, relacionadas a efetividade em estudos sobre cuidados colaborativos. Atividades colaborativas diretas são ponto positivo do apoio matricial. Cuidado estruturado e suporte em nivel organizacional devem ser encorajados. Futuros estudos devem refinar as categorias propostas e explorar seu uso para desenvolvimento do apoio matricial.

Palavras-chave Atenção primária à saúde, Serviços de saúde mental, Revisão, Cuidados colaborativos, Apoio matricial 


\section{Introdução}

Transtornos mentais são um desafio crescente dos sistemas de saúde, afetando até $25 \%$ das pessoas ao longo da vida ${ }^{1}$. O acesso a tratamentos efetivos é um problema em todos os países ${ }^{2}$. Diversos fatores contribuem para isso, como gastos públicos inadequados em saúde mental, baixa detecção, escassez de cuidados especializados $^{3,4}$. No Brasil, transtornos mentais comuns como depressão e ansiedade atingem até 35\% de adultos em estudos populacionais ${ }^{5}$ e $51-64 \%$ entre usuários da atenção primária $(\mathrm{AP})^{6}$. Mais de dois terços das pessoas afetadas por transtornos mentais graves como esquizofrenia não recebem qualquer tratamento ${ }^{7}$. Maior integração da saúde mental na atenção primária pode promover acesso a cuidados efetivos por meio de melhor detecção, tratamentos precoces, menos procedimentos desnecessários, redução do estigma, e melhor uso de recursos comunitários ${ }^{1,8}$.

Diversas estratégias de integração têm sido testadas. Treinamento de profissionais e disseminação de diretrizes podem modificar práticas individuais dos profissionais, mas são pouco efetivas para promover mudanças nos serviços ${ }^{9,10}$. Introdução de novos profissionais na AP sem uma estratégia de colaboração não parece ter impacto na melhoria do cuidado ${ }^{11}$. A consultoria de ligação, em que profissionais de saúde mental provêm suporte educacional e consultoria a outros profissionais, parece ter efeitos limitados à prescrição de medicamentos ${ }^{12}$.

Estratégias de integração com foco na colaboração entre profissionais parecem mais efetivas do que aquelas com foco em mudanças de comportamento individual ${ }^{13}$. Entre as primeiras, cuidados colaborativos (collaborative care) são amplamente usados para integrar saúde mental e AP, com evidências de efetividade no manejo de depressão, ansiedade e, em menor escala, psicoses e diabetes, quando comparados a cuidado usual ou consultoria de ligação ${ }^{12,14-17}$. Efeitos positivos incluem melhoras em: detecção, tratamento e desfechos clínicos de transtornos mentais ${ }^{15-21}$, inclusive no longo prazo $^{18}$; satisfação e qualidade de vida dos usuários ${ }^{14,21}$; e custo-efetividade, ainda que não reduza os custos totais da atenção ${ }^{17,18,21}$.

O termo 'cuidados colaborativos' abrange uma diversidade de intervenções que visam desenvolver relações de trabalho mais próximas entre profissionais para melhorar a coordenação e qualidade do cuidado ${ }^{15}$. Estas intervenções usualmente afetam os níveis individual, interprofissional e organizacional do cuidado, por exemplo ao promover redefinição de papéis profissionais ou coordenação entre serviços.

No Brasil, a principal estratégia de integração de saúde mental na AP é o apoio matricial ${ }^{22,23}$, um modelo de organização do trabalho interprofissional com objetivos e conteúdo semelhantes aos cuidados colaborativos ${ }^{24}$. $\mathrm{O}$ apoio matricial busca superar a fragmentação dos mecanismos de referência e contra-referência por meio de personalização das relações profissionais, cuidado interdisciplinar, cogestão e corresponsabilização ${ }^{23,24}$. Seus objetivos são fortalecer a AP para cuidar de problemas complexos, e ampliar o acesso a atenção especializada ${ }^{25}$. Os especialistas ("apoiadores") devem oferecer suporte educacional e assistencial aos profissionais de AP por meio de reuniões, discussão de casos, atendimentos conjuntos, atendimentos especializados ou encaminhamentos, de acordo com o caso ${ }^{26}$. O trabalho colaborativo deve ser organizado por meio de encontros para planejamento e acompanhamento de projetos terapêuticos, construção de critérios de prioridade, e definição de responsabilidades profissionais ${ }^{23,24}$.

A maior parte da produção científica sobre apoio matricial consiste de estudos descritivos ou exploratórios e se concentra na saúde mental ${ }^{27-29}$. Algumas avaliações qualitativas sugerem efeitos em detecção de transtornos mentais, definição de fluxos para tratamento, qualificação das equipes e colaboração entre profissionais ${ }^{30-32}$. Um estudo longitudinal mostrou melhora na detecção e no acompanhamento de transtornos mentais por médicos de atenção primária ${ }^{33}$. Dois estudos transversais encontraram correlação positiva entre apoio matricial e certificação das equipes de AP no Programa de Melhoria do Acesso e da Qualidade da Atenção Básica (PMAQ-AB), mas o desenho dos estudos e a diversidade de co-variáveis limita maiores inferências causais ${ }^{34,35}$. De modo geral, estudos sugerem a relevância do apoio matricial, mas há poucas evidências de efetividade, ou de que componentes se relacionam a resultados positivos.

O apoio matricial é uma intervenção complexa, com múltiplos componentes interligados exercendo efeitos em distintos níveis do siste$\mathrm{ma}^{36}$. Alguns componentes também estão presentes nos cuidados colaborativos, o que leva alguns autores a definir o apoio matricial como o modelo brasileiro de cuidados colaborativos ${ }^{24,37}$. Por exemplo, Oliveira e Campos ${ }^{24}$ apontam semelhanças entre cuidados colaborativos e apoio matricial no aspecto da colaboração interprofissional, enquanto Tesser e Poli Neto ${ }^{38}$ destacam 
convergências na coordenação da atenção e no reforço à comunicação. Porém, não encontramos artigos que comparassem de forma detalhada os componentes destes modelos, ou analizassem evidências sobre a efetividade destes. Em que pese as diferenças de cenários institucionais, o conhecimento acumulado sobre cuidados colaborativos pode ser uma fonte rica de evidências para análise do apoio matricial ${ }^{39}$. Para tal, é necessário identificar e analisar comparativamente componentes convergentes entre estes modelos.

Este artigo busca identificar, sintetizar e comparar componentes do apoio matricial e de outros modelos de cuidados colaborativos, e utilizar as evidências disponíveis sobre cuidados colaborativos, para analisar os acertos e lacunas do apoio matricial e apontar caminhos para seu desenvolvimento.

\section{Métodos}

Uma revisão narrativa da literatura foi realizada de forma a apresentar uma perspectiva ampla do tópico e provocar reflexão e debate. Este tipo de revisão mapeia o estado da arte em um tópico de pesquisa, permitindo estabelecer relações entre produções anteriores sobre o tema, identificar temáticas recorrentes e apontar novas perspecti$\operatorname{vas}^{40}$. Este método é adequado para examinar intervenções organizacionais, por discutir aspectos teórico-conceituais e do contexto de implantação de forma articulada ${ }^{41}$.

Reconhecemos a importante literatura sobre o papel do apoio matricial na democratização das organizações de saúde ${ }^{22,26}$, porém nos limitamos a considerá-lo como modelo operacional de integração de profissionais e equipes de saúde. As unidades de análise foram os componentes dos modelos, definidos como atividades e agrupados em dimensões. Como a literatura sobre cuidados colaborativos analisa principalmente intervenções para transtornos mentais, a análise apresentada se refere primariamente ao apoio matricial em saúde mental. Porém, consideramos que o artigo pode contribuir para análise do apoio matricial de forma mais abrangente.

A revisão consistiu de três etapas: (i) identificação e síntese de componentes do apoio matricial; (ii) identificação e síntese de componentes dos cuidados colaborativos e evidências de efetividade dos componentes; (iii) comparação dos componentes dos dois modelos, com análise crítica do apoio matricial à luz das evidências sobre cuidados colaborativos. No total, 61 artigos foram examinados em texto integral, dos quais 48 ( 24 sobre apoio matricial e 24 sobre cuidados colaborativos) informaram diretamente a síntese aqui apresentada (Quadro 1). Os procedimentos usados em cada etapa são detalhados a seguir.

\section{Etapa 1: Quais os componentes do apoio matricial?}

Foi realizada busca integrada no portal eletrônico periodicos.capes.gov.br, que abrange as bases eletrônicas mais relevantes para a produção científica sobre apoio matricial ${ }^{27-29,42}$. A busca foi realizada em 12/08/2017 com as palavras-chave "apoio matricial OR matriciamento" em todos os campos e reteve 286 artigos. Após análise por título e resumo, 28 trabalhos que se encaixavam nos objetivos da revisão foram lidos integralmente, dos quais 24 foram incluídos na síntese apresentada neste manuscrito (Quadro 1). Foram incluídos estudos teórico-conceituais, revisões e estudos empíricos com descrição e/ ou discussão de componentes do apoio matricial. Extratos relevantes de cada trabalho foram compilados em um documento de texto único e submetidos a uma análise temática ${ }^{43}$ simplificada, voltada especificamente para identificação e organização de componentes dos modelos (atividades e dimensões). A listagem de componentes foi consolidada conjuntamente pelos autores na forma do Quadro 2 (ver Resultados e Discussão).

\section{Etapa 2: Quais os componentes dos cuidados colaborativos, e qual a evidência da efetividade de componentes específicos?}

Uma estratégia de "bola de neve" ou snowballing, útil para revisão de tópicos onde há pouco consenso sobre terminologia e definições ${ }^{41}$, foi preferida a uma busca eletrônica por palavras-chave devido à polissemia da literatura sobre cuidados colaborativos ${ }^{15,17}$. Um pequeno número de revisões sistemáticas altamente relevantes foram identificadas a partir da base eletrônica da Biblioteca Cochrane ${ }^{12,14,15,17}$ como ponto de partida para exame de referências, levando a identificação de mais 32 trabalhos que se encaixavam nos objetivos da revisão. Ao final 36 artigos foram lidos integralmente, dos quais 24 foram incluídos na síntese aqui apresentada (Quadro 1). Foram incluídos revisões sistemáticas, narrativas, e estudos empíricos com descrição e/ou discussão de componentes dos cuidados colaborativos, com prioridade para estudos sobre efetividade de componentes específicos. De for- 


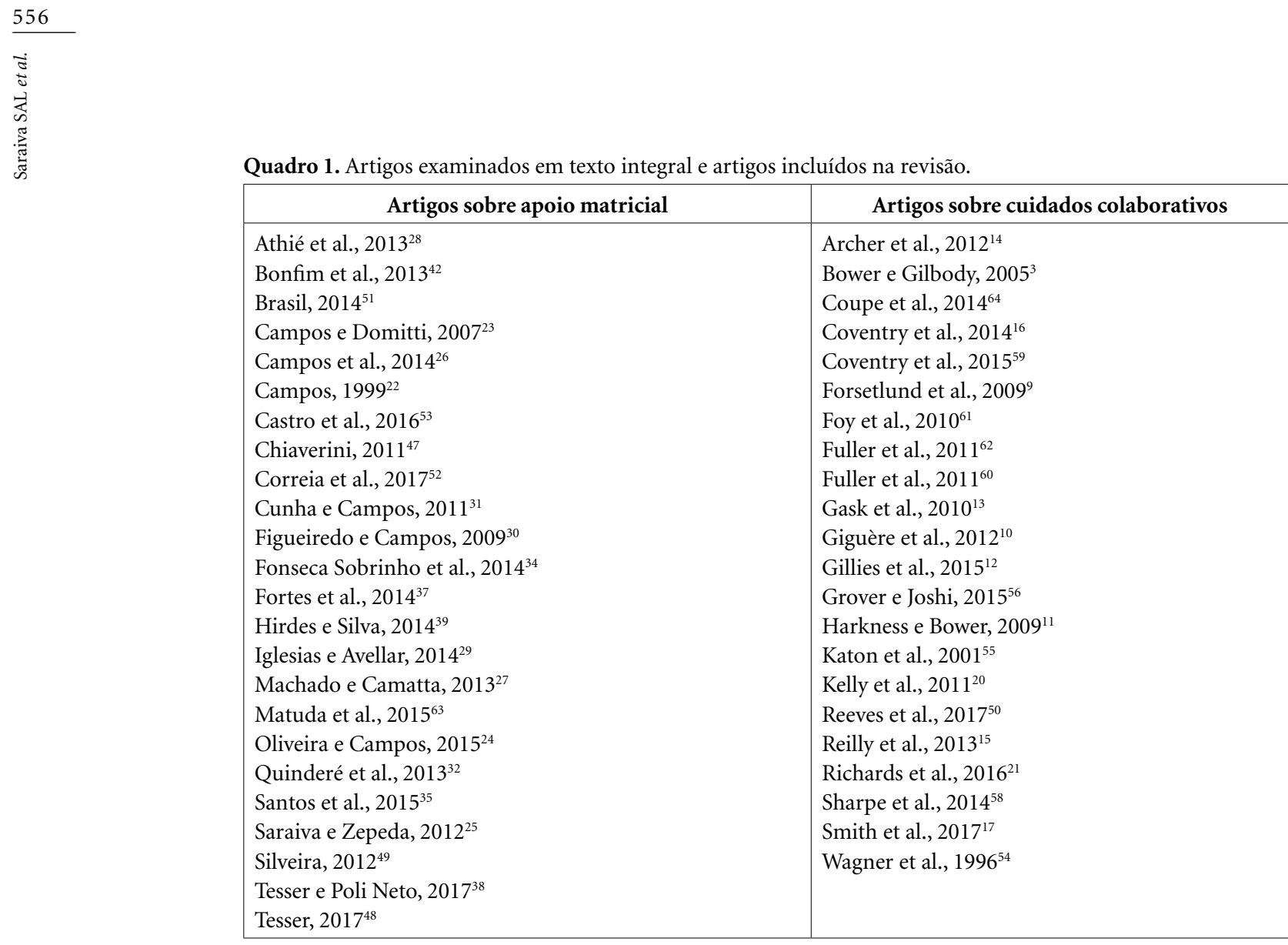

ma semelhante ao realizado para o apoio matricial, extratos dos trabalhos foram compilados em um documento de texto e submetidos a análise temática para identificação de componentes, e a listagem resultante foi consolidada conjuntamente pelos autores na forma do Quadro 3 (ver Resultados e Discussão). Adicionalmente, foram sumarizadas as evidências sobre componentes "ativos" das intervenções analisadas para subsidiar a etapa posterior de análise do apoio matricial.

\section{Etapa 3: Que lições a literatura sobre cuidados colaborativos pode oferecer para o desenvolvimento do apoio matricial?}

Após a síntese dos componentes do apoio matricial e dos cuidados colaborativos, uma análise comparativa buscou identificar semelhanças de conteúdo e/ou objetivos entre componentes do apoio matricial e componentes efetivos dos cuidados colaborativos. Em seguida, evidências sobre cuidados colaborativos foram utilizadas como evidência indireta para análise de componentes semelhantes do apoio matricial. Como resultado, foram identificadas convergências entre os dois modelos, e destacados aspectos potencialmente efetivos do apoio matricial. A comparação também possibilitou a identificação de lacunas, representadas por componentes efetivos dos cuidados colaborativos pouco desenvolvidos no apoio matricial. A análise comparativa entre os modelos encontra-se sintetizada no Quadro 4 (ver Resultados e Discussão). A análise levou em conta também a experiência dos autores ( $S$ Saraiva e J Zepeda) com a implantação do apoio matricial em Florianópolis entre 2006 e $2015^{33,44}$, principalmente na identificação de semelhanças e diferenças entre os modelos e na formulação de recomendações. $\mathrm{O}$ uso da experiência como recurso para tomada de decisões sobre intervenções em saúde é particularmente valioso quando evidências de efetividade são escassas, como no caso do apoio matricial ${ }^{45,46}$. Por fim, foi produzido um sumário de recomendações para o apoio matricial. 


\section{Resultados e discussão}

\section{Componentes do apoio matricial}

O apoio matricial é usualmente definido a partir de duas dimensões: suporte educacional ou técnico-pedagógico; e suporte assistencial, retaguarda assistencial ou cuidado especializa$\mathrm{do}^{23,24}$.

O suporte educacional é representado por ações colaborativas entre profissionais de atenção primária e especialistas, como discussão de casos, treinamentos breves, apoio à tomada de decisão, e atendimentos de usuários em conjunto. O propósito destas atividades é fortalecer as equipes de AP para lidar com problemas complexos, por meio de troca de conhecimentos e habilidades em ato. Atendimentos conjuntos podem também ser úteis para elucidar problemas e subsidiar planos terapêuticos ${ }^{26,37,47}$, o que leva alguns autores a considerá-los como atividades definidoras do apoio matricial ${ }^{37}$.

O suporte assistencial é representado pelos atendimentos especializados aos usuários. O propósito destas atividades é ampliar o acesso a cuidados especializados quando necessário ${ }^{23,26}$. Existe divergência sobre a importância desta dimensão, o que se reflete em orientações contraditórias sobre sua operacionalização ${ }^{48}$, muitas vezes subordinando-a ao suporte educacional; e em contradições entre profissionais de AP, especialistas e gestores nas experiências de implantação.
Para Tesser e Poli Neto ${ }^{38,48}$, o equilíbrio entre as dimensões assistencial e educacional é vital para o sucesso do apoio matricial, por dirimir tensões e facilitar a colaboração entre os profissionais envolvidos, contribuindo para a promoção do acesso a cuidados especializados. $\mathrm{O}$ atendimento pelo especialista é um diferencial entre o apoio matricial e outros modelos de integração como supervisão clinica e consultoria de ligação ${ }^{49}$.

$\mathrm{O}$ apoio matricial tem ainda uma dimensão de regulação, representada pela definição de diretrizes compartilhadas e critérios de atendimento, reuniões para troca de casos, e manejo de listas de espera ${ }^{23,24,26}$. Tais atividades distinguem casos para acompanhamento pela AP e especialistas, coordenam as ações de ambos e portanto facilitam o acesso a cuidado oportuno, o que leva Tesser e Poli Neto ${ }^{38}$ a defender a regulação como função estruturante do apoio matricial.

Por fim, pode-se identificar uma dimensão de cogestão, transversal e representada pela construção de acordos e decisão compartilhada sobre o trabalho. Esta dimensão traduz o propósito do apoio matricial de promover mudanças organizacionais, afetando as relações entre profissionais e os fluxos entre os serviços ${ }^{22,23}$. Gestores têm importante papel de liderança e mediação, manejando desequilíbrios de poder, atritos entre as profissões, limitação de recursos e sobrecarga de trabalho ${ }^{50}$ de forma a viabilizar a colaboração.

Alguns autores defendem ainda como tarefa do apoio matricial a articulação da AP com ou-

Quadro 2. Componentes do apoio matricial.

\begin{tabular}{|l|l|}
\hline \multicolumn{1}{|c|}{ Dimensões } & \multicolumn{1}{|c|}{ Atividades } \\
\hline $\begin{array}{l}\text { Suporte educacional } \\
\text { Interação entre especialistas e profissionais de } \\
\text { AP com objetivo de fortalecer a capacidade } \\
\text { assistencial da AP }\end{array}$ & $\begin{array}{l}\text { Discussão de casos e interconsulta (inclusive tele-apoio); } \\
\text { Construção e seguimento de planos terapêuticos; } \\
\text { Provisão de capacitação e recursos educacionais; } \\
\text { Atendimentos conjuntos (consultas, visitas, grupos); } \\
\text { Apoio a tomada de decisão em situações urgentes. }\end{array}$ \\
\hline $\begin{array}{l}\text { Cuidado especializado } \\
\text { Interação entre especialistas e usuários com } \\
\text { objetivo de ampliar o acesso destes a cuidado } \\
\text { especializado oportuno }\end{array}$ & $\begin{array}{l}\text { Atendimentos especializados (consultas, visitas e grupos); } \\
\text { Discussão de casos com outros serviços; } \\
\text { Encaminhamentos pra outros serviços (inclusive em } \\
\text { situações urgentes). }\end{array}$ \\
\hline $\begin{array}{l}\text { Regulação } \\
\text { Interação entre especialistas e profissionais } \\
\text { de AP com objetivo de coordenar as ações } \\
\text { oferecidas aos usuários }\end{array}$ & $\begin{array}{l}\text { Gestão de listas de espera, referência e devolução de casos; } \\
\text { Construção de diretrizes e fluxos de atenção (inclusive para } \\
\text { outros serviços); } \\
\text { Critérios de prioridade para atendimento especializado. }\end{array}$ \\
\hline $\begin{array}{l}\text { Cogestão } \\
\text { Interação entre especialistas e profissionais de } \\
\text { AP com objetivo de fortalecer a colaboração } \\
\text { entre profissionais e serviços envolvidos }\end{array}$ & $\begin{array}{l}\text { Definição conjunta de diretrizes para o trabalho; } \\
\text { Reuniões regulares para gestão do trabalho compartilhado; } \\
\text { Definição de papéis profissionais no cuidado compartilhado; } \\
\text { Definç̧ão de necessidades de capacitação. }\end{array}$ \\
\hline
\end{tabular}

Fonte: Elaborado pelos autores, com base na revisão feita para este artigo. 


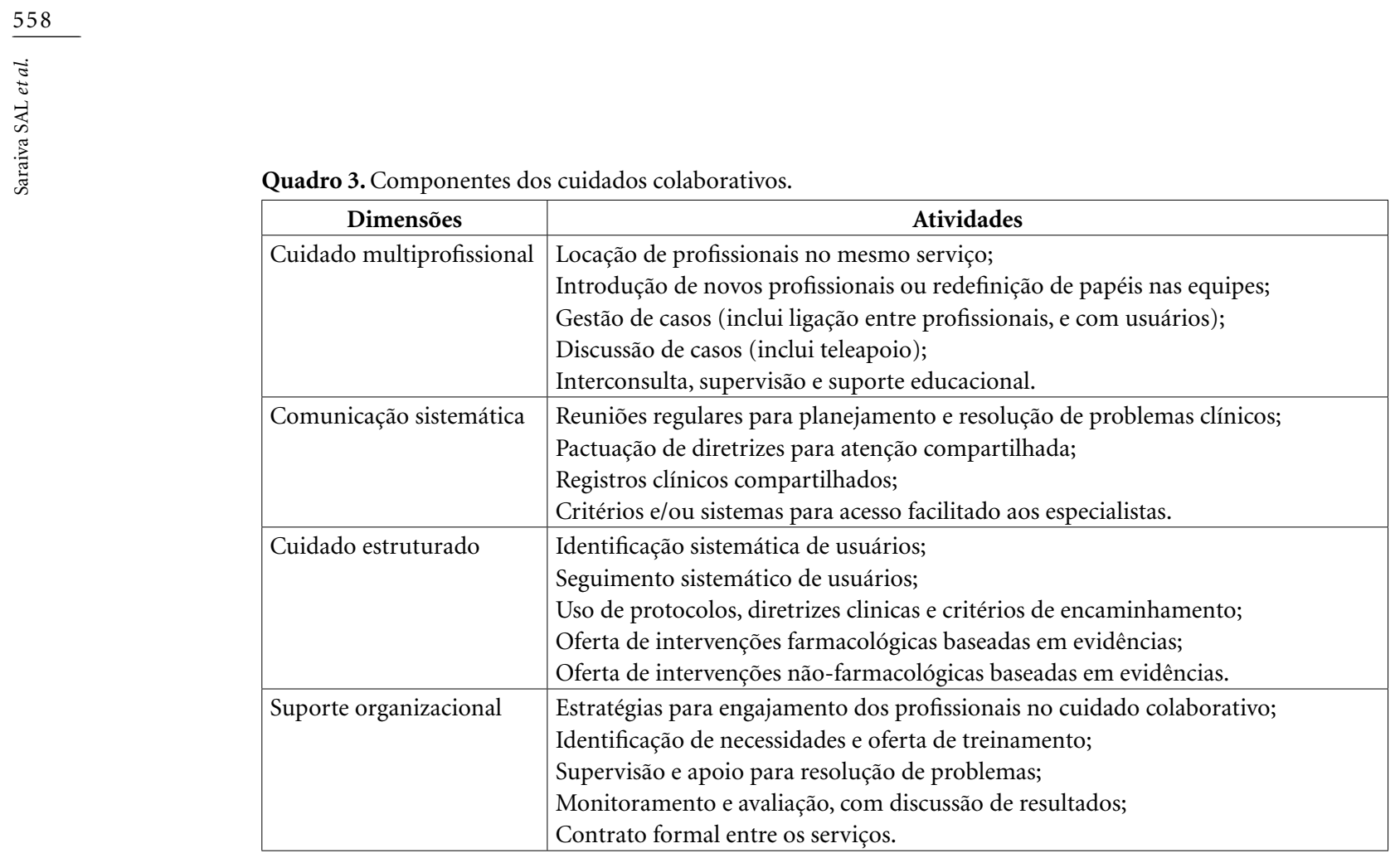

Fonte: Elaborado pelos autores, com base na revisão feita para este artigo.

tros serviços de saúde, sociais e comunitários, configurando redes de cuidados $^{37}$, particularmente na proposta dos NASF ${ }^{51,52}$. Porém, a indefinição de escopo e prioridades deixa margem para superposição de tarefas entre especialistas e profissionais de $\mathrm{AP}^{48}$. Por este motivo, neste artigo incluímos apenas atividades claramente delimitadas como definição de diretrizes de atenção e encaminhamento entre serviços.

Outras classificações de componentes do apoio matricial adotam critérios distintos dos nossos, por exemplo, de acordo com os atores envolvidos - ações conjuntas com usuários, ações conjuntas sem usuários e ações do especialista com usuários ${ }^{48}$ - ou com as ferramentas utilizadas - por ex., visitas conjuntas, discussão de caso, teleapoio ${ }^{35,53}$. Porém, o conteúdo da intervenção mostra-se relativamente constante.

Em síntese, definimos quatro dimensões para o apoio matricial - suporte educacional, cuidado especializado, regulação, e uma dimensão transversal de cogestão. As atividades identificadas foram agrupadas nestas dimensões a partir do seu objetivo primário. O Quadro 2 sintetiza os componentes do apoio matricial.

\section{Componentes dos cuidados colaborativos}

$\mathrm{Na}$ literatura de língua inglesa, shared care (cuidado compartilhado), collaborative care (cui- dado colaborativo) e integrated care (cuidado integrado) são usados de maneira intercambiável para se referir ora a uma mesma intervenção, ora a níveis de integração entre serviços ${ }^{15,17}$. Neste artigo, incluímos modelos de colaboração entre profissionais de atenção primária e especialistas, independentemente da denominação, desde que não restritos apenas a supervisão, encaminhamentos ou treinamento, e utilizamos cuidados colaborativos para nos referir ao conjunto dos modelos analisados.

Nos cuidados colaborativos, pelo menos dois profissionais de distintas disciplinas devem estar envolvidos no cuidados aos usuários ${ }^{12,17}$, podendo ou não haver outros com papéis de ligação ${ }^{16,18}$. A colaboração é organizada por meio de atividades de ligação, gestão conjunta de casos, reuniões entre as equipes, ou sistemas de comunicação ${ }^{15,17,20}$. Estes modelos são inspirados no manejo de doenças crônicas, com forte presença de cuidado estruturado por meio de protocolos e diretrizes ${ }^{54-56}$.

Uma definição amplamente aceita de cuidados colaborativos ${ }^{57}$ exige a presença de quatro componentes: (i) cuidado multiprofissional (como gestão de casos); (ii) cuidados estruturados (como uso de protocolos); (iii) acompanhamento sistemático (como seguimento regular de usuários); e (iv) reforço à comunicação (como reuniões e discussões de caso). Categorias seme- 
Quadro 4. Comparação entre componentes do apoio matricial e dos cuidados colaborativos.

\begin{tabular}{|c|c|}
\hline Componentes do apoio matricial & $\begin{array}{c}\text { Componentes semelhantes dos cuidados colaborativos e } \\
\text { evidências de efetividade }\end{array}$ \\
\hline \multicolumn{2}{|l|}{ Dimensão: Suporte educacional } \\
\hline Discussão de casos e interconsulta & \multirow{2}{*}{$\begin{array}{l}\text { Discussão de casos; Interconsulta, supervisão e suporte educacional. } \\
\text { Efetivos como oportunidades de colaboração direta e comunicação } \\
\text { interativa }^{50,60,61} \text {. }\end{array}$} \\
\hline $\begin{array}{l}\text { Provisão de capacitação e recursos } \\
\text { educacionais }\end{array}$ & \\
\hline $\begin{array}{l}\text { Construção e seguimento de planos } \\
\text { terapêuticos }\end{array}$ & $\begin{array}{l}\text { Gestão de casos; Reuniões para planejamento e resolução de } \\
\text { problemas clínicos; Seguimento sistemático de usuários. } \\
\text { Efetivo pelos aspectos de comunicação interativa }{ }^{50,60,61} \text { e/ou de oferta } \\
\text { de cuidados estruturados }{ }^{16,20} \text {. }\end{array}$ \\
\hline Atendimentos conjuntos & $\begin{array}{l}\text { Não explícitos nos cuidados colaborativos, porém são exemplos de } \\
\text { atividades colaborativas diretas. } \\
\text { Efetivos como exemplos de atividades colaborativas diretas }{ }^{50,61} \text {. }\end{array}$ \\
\hline $\begin{array}{l}\text { Apoio a tomada de decisão em situações } \\
\text { urgentes }\end{array}$ & $\begin{array}{l}\text { Interconsulta, supervisão e suporte educacional (do qual podem ser } \\
\text { atividades específicas). } \\
\text { Efetivos como oportunidade de colaboração direta e comunicação } \\
\text { interativa }^{50,60,61} \text {. }\end{array}$ \\
\hline \multicolumn{2}{|l|}{ Dimensão: Cuidado especializado } \\
\hline Atendimentos especializados & $\begin{array}{l}\text { Oferta de intervenções farmacológicas e não-farmacológicas. } \\
\text { Efetivos se forem parte de cuidados estruturados baseados em } \\
\text { evidências clínicas }{ }^{14,16,17,20} \text {. }\end{array}$ \\
\hline Discussão de casos com outros serviços & \multirow{2}{*}{$\begin{array}{l}\text { Uso de protocolos, diretrizes clínicas e critérios de encaminhamento } \\
\text { (do qual podem ser atividades específicas). } \\
\text { Efetivos se forem parte de cuidados estruturados baseados em } \\
\text { evidências clínicas }{ }^{14,16,17,20} \text {. }\end{array}$} \\
\hline Encaminhamentos para outros serviços & \\
\hline \multicolumn{2}{|l|}{ Dimensão: Regulação } \\
\hline $\begin{array}{l}\text { Gestão de listas de espera, referência e } \\
\text { devolução de casos }\end{array}$ & $\begin{array}{l}\text { Reuniões para planejamento e resolução de problemas clínicos (do } \\
\text { qual podem ser atividades específicas). } \\
\text { Potencialmente efetivos pela oportunidade de comunicação } \\
\text { interativa entre os profissionais }\end{array}$ \\
\hline $\begin{array}{l}\text { Construção de diretrizes e fluxos de } \\
\text { atenção }\end{array}$ & $\begin{array}{l}\text { Pactuação de diretrizes para atenção compartilhada (os modelos } \\
\text { enfatizam aspectos distintos da atividade). } \\
\text { Potencialmente efetivo pelo aspecto de comunicação interativa } \\
\text { e/ou se implicar em oferta de cuidados estruturados } \\
\text { e/,16,17,20. }\end{array}$ \\
\hline $\begin{array}{l}\text { Critérios de prioridade para } \\
\text { atendimento especializado }\end{array}$ & $\begin{array}{l}\text { Critérios e/ou sistemas para acesso facilitado aos especialistas; Uso } \\
\text { de protocolos, diretrizes clinicas e critérios de encaminhamento } \\
\text { (pouco enfatizado no apoio matricial). } \\
\text { Potencialmente efetivo como exemplo de oferta de cuidado } \\
\text { estruturado }{ }^{14,16,17,20} \text {. }\end{array}$ \\
\hline \multicolumn{2}{|l|}{ Dimensão: Cogestão } \\
\hline $\begin{array}{l}\text { Definição conjunta de diretrizes de } \\
\text { trabalho }\end{array}$ & $\begin{array}{l}\text { Pactuação de diretrizes para atenção compartilhada. } \\
\text { Efetivo pelos aspectos de comunicação interativa }{ }^{50,60,61} \text { e estruturação } \\
\text { do cuidado }{ }^{14,16,17,20} \text {. }\end{array}$ \\
\hline $\begin{array}{l}\text { Reuniões regulares para gestão do } \\
\text { trabalho }\end{array}$ & $\begin{array}{l}\text { Reuniões para planejamento e resolução de problemas clínicos. } \\
\text { Efetivo se oferecer oportunidade de comunicação interativa sobre } \\
\text { problemas clínicos }{ }^{50,60,61} \text {. }\end{array}$ \\
\hline $\begin{array}{l}\text { Definição de papéis profissionais no } \\
\text { cuidado }\end{array}$ & $\begin{array}{l}\text { Introdução de novos profissionais ou redefinição de papéis; } \\
\text { Pactuação de diretrizes para atenção compartilhada. } \\
\text { Efetivo pelo aspecto de envolvimento dos profissionais na definição } \\
\text { de papéis }{ }^{20,60,62} \text {. }\end{array}$ \\
\hline Definição de necessidades de capacitação & $\begin{array}{l}\text { Identificação de necessidades e oferta de treinamento. } \\
\text { Efetivo como parte de uma estratégia de suporte organizacional }{ }^{20,62} \text {. }\end{array}$ \\
\hline
\end{tabular}

Fonte: Elaborado pelos autores, com base na revisão feita para este artigo. 
lhantes foram propostas por Bower et al. ${ }^{18}$ : (i) mecanismos de ligação entre os profissionais; (ii) oferta estruturada de intervenções; (iii) compartilhamento de informação sobre os usuários. Uma síntese detalhada que identificou 14 componentes de cuidados colaborativos em saúde mental manteve coerência com estas categorias gerais, mostrando sua relevância e consistência ${ }^{15}$.

Algumas revisões apontam ainda componentes que podem ser definidos como suporte organizacional ao trabalho colaborativo ${ }^{15,17,20}$, abrangendo estratégias de envolvimento sistemático, treinamento, supervisão e apoio aos profissionais, além de monitoramento e avaliação dos resultados do trabalho colaborativo.

Apesar da repetição de componentes entre as revisões, há variação em relação ao que é oferecido de fato ${ }^{14}$, algo que deve ser levada em conta ao se extrapolar resultados dos estudos para outros contextos ${ }^{58}$. Por exemplo, a gestão de casos, presente na maior parte dos modelos de cuidados colaborativos, pode ser realizada por um profissional especificamente treinado para $\mathrm{tal}^{18,21}$ ou pelos próprios enfermeiros das equipes de $\mathrm{AP}^{16,59}$, e pode variar de atividades de ligação entre os profissionais ou com os pacientes, como telefonemas, até participação direta na assistência. Enfermeiros ou outros profissionais parecem ser igualmente efetivos ${ }^{16}$, demonstrando a viabilidade de introduzir mudanças no cuidado sem alterar significativamente os recursos disponibilizados.

Cuidado multiprofissional e comunicação sistemática se relacionam a maior efetividade do trabalho colaborativo em saúde, principalmente quando representados por atividades colaborativas diretas entre profissionais ${ }^{60}$. Duas revisões sistemáticas sobre colaboração interprofissional ${ }^{50,61}$ mostram efeitos positivos (em processos clínicos e resultados de saúde) de atividades que promoveram interação e negociação entre profissionais, como reuniões, discussão por telefone ou meios eletrônicos, ou atendimento conjunto de usuários. Estes achados são corroborados por revisões sobre cuidados colaborativos em saúde mental , que destacam a importância de atividades colaborativas como gestão de casos, interconsulta, suporte regular pelo especialista, e pactuação de diretrizes locais de atenção ${ }^{60}$.

Alguns componentes de cuidado estruturado, como identificação sistemática de usuários, uso de protocolos de tratamento escalonado, e seguimento sistemático dos usuários parecem particularmente efetivos para melhora clínica de depressão e ansiedade ${ }^{20,16}$. Resultados distintos foram observados nos cuidados colaborativos para psicoses, em que cuidado multiprofissional e comunicação sistemática, por exemplo por reuniões regulares e gestão de casos, parecem mais importantes do que estruturação do cuidado ${ }^{20}$. Estes achados sugerem a importância de considerar variações nos cuidados colaborativos para distintas condições.

Por fim, estratégias de suporte à integração parecem especialmente relevantes para o sucesso do trabalho colaborativo: apoio organizacional, representado por atividades como engajamento, treinamento, supervisão e discussão de resultados com profissionais ${ }^{20}$; desenvolvimento de processos para comunicação estruturada entre os profissionais, para planejamento e resolução de problemas clínicos e construção de diretrizes e critérios comuns $^{62}$; e definição clara de papéis profissionais ${ }^{60}$. Por outro lado, contratos formais entre os serviços não parecem melhorar a integração ${ }^{60}$, sugerindo que afetar o trabalho clínico dos profissionais pode ser mais relevante do que estabelecer acordos no nível gerencial dos serviços.

Em síntese, identificamos quatro dimensões dos cuidados colaborativos - cuidado multiprofissional, comunicação sistemática, cuidado estruturado, e suporte organizacional à colaboração, apresentadas no Quadro 3. Dada a polissemia e diversidade da literatura sobre cuidados colaborativos, esta síntese deve ser interpretada com mais cautela do que aquela referente ao apoio matricial. Porém, ainda que provisórias, algumas inferências podem ser úteis para análise do apoio matricial, conforme descrito a seguir.

\section{Convergências entre cuidados colaborativos e apoio matricial}

O Quadro 4 sintetiza as convergências entre apoio matricial e cuidados colaborativos e aponta aspectos dos componentes do apoio matricial potencialmente relacionados a maior efetividade, com base em evidências sobre cuidados colaborativos. A semelhança mais evidente entre os modelos está nas atividades de interação direta entre profissionais presentes na dimensão de suporte educacional do apoio matricial e nas dimensões de cuidado multiprofissional e comunicação sistemática dos cuidados colaborativos, e representadas por atividades como discussão de casos, construção de planos terapêuticos, e provisão de suporte educacional. O apoio a tomada de decisão em situações urgentes pode ser visto como uma forma de interconsulta. Os atendimentos conjuntos, presentes de forma explícita apenas 
no apoio matricial, também podem ser considerados oportunidades de colaboração direta entre os profissionais.

Correspondência indireta pode ser traçada entre as dimensões de cuidado especializado do apoio matricial e cuidado estruturado dos cuidados colaborativos. Os cuidados colaborativos enfatizam a oferta sistemática de intervenções estruturadas para determinadas condições, usualmente com base em protocolos, diretrizes e critérios de atenção, enquanto o apoio matricial evita recomendações prescritivas sobre o conteúdo da atenção. Por outro lado, um dos objetivos do apoio matricial é melhorar a qualidade da atenção, e uma das estratégias para tal é a construção de diretrizes locais de atenção - o que não é incompatível com uma maior oferta de ações comprovadamente efetivas.

A semelhança é mais superficial na dimensão de regulação do apoio matricial. Atividades como construção de diretrizes de atenção e critérios de prioridade para atendimento especializado, apesar de presentes em ambos modelos, assumem caráter ordenador do trabalho nos cuidados colaborativos, enquanto o apoio matricial enfatiza o aspecto de interação pessoal e negociação entre os profissionais envolvidos nestas atividades.

Por fim, há semelhanças entre a dimensão de cogestão do apoio matricial e atividades das dimensões de comunicação sistemática e suporte organizacional dos cuidados compartilhados, como reuniões regulares, definição de papéis profissionais e identificação de necessidades de capacitação.

O padrão de semelhanças e diferenças é coerente com a origem de cada modelo. Cuidados colaborativos visam melhorar o cuidado de condições específicas, o que se reflete na ênfase em estruturação do cuidado, enquanto o apoio matricial visa reformar as relações de trabalho em saúde, o que se reflete na ênfase em interação direta e cogestão. Esta diferença é destacada por Oliveira e Campos ${ }^{24}$, que apontam a ênfase no aspecto político da clínica e na democratização das relações entre os profissionais como exemplos de avanço do apoio matricial em relação aos cuidados colaborativos.

\section{Lições dos cuidados colaborativos para o apoio matricial}

Atividades colaborativas diretas que oportunizem comunicação sistemática e interativa têm evidência de efetividade nos cuidados colaborativos e devem ser fortalecidas no apoio matricial. Exemplos destas atividades são: reuniões presenciais ou discussão de casos por outros meios; atendimentos conjuntos; construção de planos terapêuticos, gestão de casos e resolução de problemas clínicos; e pactuação de diretrizes de atenção e papéis profissionais ${ }^{17,20,50,57,60-62}$.

Maior estruturação do cuidado colaborativo, com identificação e seguimento sistemático de usuários, uso de protocolos e critérios de encaminhamento, e oferta sistemática de intervenções baseadas em evidencias, pode melhorar os resultados em saúde para algumas condições como depressão e ansiedade ${ }^{14,16,17,20}$. A incorporação destes elementos ao apoio matricial, se realizada com pactuação local entre os profissionais, poderia contribuir para a efetividade clínica do cuidado e ao mesmo tempo reforçar a colaboração e comunicação entre os profissionais envolvidos $^{23,24,26}$.

Por fim, maior apoio organizacional à colaboração, por meio de envolvimento sistemático dos profissionais, apoio para resolução de problemas, monitoramento e discussão de resultados, poderia levar a uma melhor integração e melhores resultados de saúde ${ }^{20,62}$. A colaboração interprofissional não pode depender do esforço pessoal dos trabalhadores em saúde, devendo contar com recursos organizacionais de apoio ao trabalho compartilhado ${ }^{25}$, geralmente insuficientes no SUS ${ }^{63}$.

\section{Limitações do estudo}

Este artigo apresenta algumas limitações. A primeira diz respeito à complexidade das intervenções analisadas, diversidade da literatura e dificuldade de comparação dos modelos. A falta de consenso sobre componentes do apoio matricial e dos cuidados colaborativos e as variações na implantação dificultam a síntese do conhecimento existente. Adicionalmente, o fato de os estudos sobre cuidados colaborativos serem via de regra voltados para condições específicas, em contexto de pesquisa, e em países desenvolvidos, limita a extrapolação dos achados para o apoio matricial, implantado de forma não-experimental, não seletiva, num sistema de saúde com recursos mais limitados. A segunda limitação diz respeito ao método: não foi realizada uma revisão sistemática, e é possível que estudos relevantes não tenham sido incluídos, levando a interpretações parciais. No entanto, o caráter exploratório do presente estudo justifica a metodologia empregada, e futuros estudos poderão aperfeiçoar e testar as categorias aqui propostas possibilitando me- 
lhor uso da literatura para desenvolvimento do apoio matricial.

\section{Conclusão}

O apoio matricial é a principal estratégia brasileira de integração de novos profissionais na atenção primária, e análise sistemática da literatura existente sobre outros modelos de colaboração pode fornecer subsídios para seu desenvolvimento. A síntese aqui apresentada avança em relação a trabalhos anteriores ao apresentar pela primeira vez uma análise sistemática e comparativa de componentes do apoio matricial e dos cuidados colaborativos. Nossa síntese aponta acertos do apoio matricial, como a ênfase na interação direta e comunicação sistemática entre os profissionais com foco em problemas clínicos; aspectos importantes de sua implantação que necessitam maior atenção, como o suporte organizacional ao trabalho colaborativo; e potenciais direções para seu desenvolvimento, como a incorporação de intervenções estruturadas para algumas condições, com base em diretrizes acordadas localmente. Estas linhas temáticas e a síntese de componentes aqui apresentada podem servir de base para futuras iniciativas e estudos de implantação e avaliação do apoio matricial.

\section{Colaboradores}

SAL Saraiva participou da concepção do artigo, revisão, análise e interpretação da literatura e redação do artigo. J Zepeda participou da concepção do artigo, revisão, análise e interpretação da literatura e redação do artigo. AF Liria participou da concepção, redação e revisão crítica do artigo. 


\section{Referências}

1. World Health Organization (WHO), World Organization of Family Doctors (WONCA). Integrating mental health into primary care: a global perspective. Genebra, Londres: WHO, WONCA; 2008.

2. World Health Organization (WHO). Mental Health Action Plan 2013-2020. Genebra: WHO; 2013.

3. Bower P, Gilbody S. Managing common mental health disorders in primary care: conceptual models and evidence base. BMJ 2005; 330(7495):839-842.

4. Saxena S, Sharan P, Garrido M, Saraceno B. World Health Organization's Mental Health Atlas 2005: implications for policy development. World Psychiatry 2006; 5(3):179-184.

5. Maragno L, Goldbaum M, Gianini RJ, Novaes HMD, César CLG. Prevalência de transtornos mentais comuns em populações atendidas pelo Programa Saúde da Família (QUALIS) no Município de São Paulo, Brasil. Cad Saude Publica 2006; 22(8):1639-1648.

6. Gonçalves DA, Mari JJ, Bower P, Gask L, Dowrick C, Tófoli LF, Campos M, Portugal FB, Ballester D, Fortes S. Brazilian multicentre study of common mental disorders in primary care: rates and related social and demographic factors. Cad Saude Publica 2014; 30(3):623-632.

7. Andrade LH, Wang YP, Andreoni S, Silveira CM, Alexandrino-Silva C, Siu ER, Nishimura R, Anthony JC, Gattaz WF, Kessler RC. Mental disorders in megacities: findings from the São Paulo megacity mental health survey, Brazil. PLoS One 2012; 7(2):e31879.

8. World Health Organization (WHO). Scaling up care for mental, neurological, and substance use disorders. Genebra: WHO; 2008

9. Forsetlund L, Bjorndal A, Rashidian A, Jamtvedt G, O'Brien MA, Wolf F, Davis D, Odgaard-Jensen J, Oxman AD. Continuing education meetings and workshops: effects on professional practice and health care outcomes. Cochrane Database Syst Rev 2009; 2:CD003030.

10. Giguere A, Legare F, Grimshaw J, Turcotte S, Fiander M, Grudniewicz A, Makosso-Kallyth S, Wolf FM, Farmer AP, Gagnon MP. Printed educational materials: effects on professional practice and healthcare outcomes. Cochrane Database Syst Rev 2012; 10:CD004398.

11. Harkness EF, Bower PJ, Harkness, Elaine F, Bower, Peter J. On-site mental health workers delivering psychological therapy and psychosocial interventions to patients in primary care: effects on the professional practice of primary care providers. Cochrane Database Syst Rev 2009; 1:CD000532.

12. Gillies D, Buyck P, Parker AG, Hetrick SE. Consultation liaison in primary care for people with mental disorders. Cochrane Database Syst Rev 2015; 9:CD007193.

13. Gask L, Bower P, Lovell K, Escott D, Archer J, Gilbody S, Lankshear AJ, Simpson AE, Richards DA. What work has to be done to implement collaborative care for depression? Process evaluation of a trial utilizing the Normalization Process Model. Implement Sci 2010; 5(1):15.

14. Archer J, Bower P, Gilbody S, Lovell K, Richards D, Gask L, Dickens C, Coventry P. Collaborative care for depression and anxiety problems. Cochrane Database Syst Rev 2012; 10:CD006525.
15. Reilly S, Planner C, Gask L, Hann M, Knowles S, Druss B, Lester H. Collaborative care approaches for people with severe mental illness. Cochrane Database Syst Rev 2013; 11:CD009531.

16. Coventry PA, Hudson JL, Kontopantelis E, Archer J, Richards DA, Gilbody S, Dickens C, Gask L, Waheed W, Bower P. Characteristics of effective collaborative care for treatment of depression: A systematic review and meta-regression of 74 randomised controlled trials. PLoS One 2014; 9(9):e108114.

17. Smith S, Cousins G, Clyne B, Allwright S, Dowd OT. Shared care across the interface between primary and specialty care in management of long term conditions. Cochrane Database Syst Rev 2017; 2:CD004910.

18. Bower P, Gilbody S, Richards D, Fletcher J, Sutton A. Collaborative care for depression in primary care. Making sense of a complex intervention: systematic review and meta-regression. Br J Psychiatry 2006; 189:484-493.

19. Christensen H, Griffiths KM, Gulliver A, Clack D, Kljakovic M, Wells L. Models in the delivery of depression care: a systematic review of randomised and controlled intervention trials. BMC Fam Pract 2008; 9(1):25.

20. Kelly BJ, Perkins DA, Fuller JD, Parker SM. Shared care in mental illness: A rapid review to inform implementation. Int J Ment Health Syst 2011; 5:31.

21. Richards DA, Bower P, Chew-Graham C, Gask L, Lovell K, Cape J, Pilling S, Araya R, Kessler D, Barkham M, Bland JM, Gilbody S, Green C, Lewis G, Manning C, Kontopantelis E, Hill JJ, Hughes-Morley A, Russel A. Clinical effectiveness and cost-effectiveness of collaborative care for depression in UK primary care (CADET): a cluster randomised controlled trial. $\mathrm{He}$ alth Technol Assess 2016; 20(14):1-192.

22. Campos GW. Equipes de referência e apoio especializado matricial: um ensaio sobre a reorganização do trabalho em saúde. Cien Saude Colet 1999; 4(2):393403.

23. Campos GW, Domitti AC. Apoio matricial e equipe de referência: uma metodologia para gestão do trabalho interdisciplinar em saúde. Cad Saude Publica 2007; 23(2):399-407.

24. Oliveira MM, Campos GWS. Matrix support and institutional support: analyzing their construction. Cien Saude Colet 2015; 20(1):229-238.

25. Saraiva S, Zepeda J. Princípios do Apoio Matricial. In: Gusso G, Lopes J, editores. Tratado de Medicina de Família e Comunidade. $1^{\text {a }}$ ed. Porto Alegre: Artmed; 2012.

26. Campos GW, Figueiredo MD, Pereira N, Castro CP. A aplicação da metodologia Paideia no apoio institucional, no apoio matricial e na clínica ampliada. Interface (Botucatu) 2014; 18:983-995.

27. Machado DK, Camatta MW. Apoio matricial como ferramenta de articulação entre a Saúde Mental e a Atenção Primária à Saúde. Cad Saude Colet 2013; 21(2):224-232.

28. Athié K, Fortes S, Delgado PGG. Matriciamento em saúde mental na Atenção Primária: uma revisão crítica (2000-2010). Rev Bras Med Família e Comunidade 2013; 8(26):64-74. 
29. Iglesias A, Avellar LZ. Apoio Matricial: um estudo bibliográfico. Cien Saude Colet 2014; 19(9):3791-3798.

30. Figueiredo MD, Campos RO. Saúde Mental na atenção básica à saúde de Campinas, SP: uma rede ou um emaranhado? Cien Saude Colet 2009; 14(1):129-138.

31. Cunha GT, Campos GW. Apoio matricial e atenção primária em saúde. Saude e Soc. 2011; 20(4):961-970.

32. Quinderé PHD, Jorge MSB, Nogueira MSL, Costa LFA, Vasconcelos MGF. Acessibilidade e resolubilidade da assistência em saúde mental: a experiência do apoio matricial. Cien Saude Colet 2013; 18(7):21572166.

33. Saraiva S. La integración entre salud mental y atención primaria: efectos de la implantación de la atención compartida (apoyo matricial) en Florianópolis, Brasil [tese]. Madrid: Universidad de Alcalá; 2015.

34. Fonseca Sobrinho D, Machado AT, Lima AM, Jorge AO, Reis CMR, Abreu DMX, Araújo LHL, Evangelista MLF, Escobar AL, Santos CRI, Santos AF. Compreendendo o Apoio Matricial e o resultado da certificação de qualidade nas áreas de atenção à criança, mulher, diabetes/hipertensão e saúde mental. Saude Debate 2014; 38(esp.):83-93.

35. Santos AF, Machado AT, Reis CMR, Abreu DMX, Araújo LHL, Rodrigues SC, Lima ÂM, Jorge AO, Fonseca Sobrinho D. Institutional and matrix support and its relationship with primary healthcare. Rev Saude Publica 2015; 49:54.

36. Moore GF, Audrey S, Barker M, Bond L, Bonell C, Hardeman W, Moore L, O'Cathain A, Tinati T, Wight D, Baird J. Process evaluation of complex interventions: Medical Research Council guidance. BMJ 2015; 350:h1258.

37. Fortes S, Menezes A, Athié K, Chazan LF, Rocha H, Thiesen J, Ragoni C, Pithon T, Machado A. Psiquiatria no século XXI: transformações a partir da integração com a atenção primária pelo matriciamento. Physis 2014; 24(4):1079-1102.

38. CD, Poli Neto P. Atenção especializada ambulatorial no Sistema Único de Saúde: para superar um vazio. Cien Saude Colet 2017; 22(3):941-951.

39. Hirdes A, Silva MKR. Apoio matricial: um caminho para a integração saúde mental e atenção primária. Saude Debate 2014; 38(102):582-592.

40. Vosgerau DSR, Romanowski JP. Estudos de revisão: implicações conceituais e metodológicas. Rev Dialogo Educ 2014; 14(474):165.

41. Green BN, Johnson CD, Adams A. Writing narrative literature reviews for peer-reviewed $\backslash$ njournals: secrets of the trade. J Chiropr Med 2006; 5(3):101-117.

42. Bonfim IG, Bastos ENE, Góis CWL, Tófoli LF. Apoio matricial em saúde mental na atenção primária à saúde: uma análise da produção científica e documental. Interface (Botucatu) 2013; 17(45):287-300.

43. Braun V, Clarke V. Using thematic analysis in psychology. Qual Res Psychol 2006; 3(2):77-101.

44. Saraiva S, Cremonese E. Implantação do modelo de apoio matricial em saúde mental no município de Florianópolis, SC. In: III Concurso nacional de experiências em saúde da família: trabalhos premiados. Brasília: MS; 2008. p. 37-48.
45. Patel V, Araya R, Chatterjee S, Chisholm D, Cohen A, De Silva M, Hosman C, McGuire H, Rojas G, van Ommeren M. Treatment and prevention of mental disorders in low-income and middle-income countries. Lancet 2007; 370(9591):991-1005.

46. Araya R. Experience counts [commentarie]. World Psychiatry 2008; 7:93-94.

47. Chiaverini DH, organizadora. Guia prático de matriciamento em saúde mental. Brasília: MS: Centro de Estudos e Pesquisa em Saúde Coletiva; 2011.

48. Tesser CD. Núcleos de Apoio à Saúde da Família, seus potenciais e entraves: uma interpretação a partir da atenção primária à saúde. Interface (Botucatu) 2017; 21(62):565-578.

49. Silveira ER. Práticas que integram a saúde mental à saúde pública: o apoio matricial e a interconsulta. Cien Saude Colet 2012; 17(9):2377-2386.

50. Reeves S, Pelone F, Harrison R, Goldman J, Zwarenstein $M$. Interprofessional collaboration to improve professional practice and healthcare outcomes. Cochrane Database Syst Rev 2017;6:CD000072.

51. Ministério da Saúde (MS). Cadernos de Atenção Básica: Núcleo de Apoio à Saúde da Família: Ferramentas para a gestão e para o trabalho cotidiano. Brasília: MS; 2014. (Cadernos de Atenção Básica, no 39).

52. Correia PCI, Goulart PM, Furtado JP. A avaliabilidade dos Núcleos de Apoio à Saúde da Família (NASF). Saude Debate 2017; 41(Supl.):345-359.

53. Castro CP, Oliveira MM, Campos GW. Apoio Matricial no SUS Campinas: análise da consolidação de uma prática interprofissional na rede de saúde. Cien Saude Colet 2016; 21(5):1625-1636.

54. Wagner EH, Austin BT, Von Korff M. Organizing care for patients with chronic illness. Milbank Q 1996; 74(4):511-544.

55. Katon W, Von Korff M, Lin E, Simon G. Rethinking practitioner roles in chronic illness: the specialist, primary care physician, and the practice nurse. Gen Hosp Psychiatry 2001; 23(3):138-144.

56. Grover A, Joshi A. An Overview of Chronic Disease Models: A Systematic Literature Review. Glob J Health Sci 2015; 7(2):210-227.

57. Gunn J, Diggens J, Hegarty K, Blashki G. A systematic review of complex system interventions designed to increase recovery from depression in primary care. BMC Health Serv Res 2006; 6:88.

58. Sharpe M. Not all forms of collaborative care are the same [letter]. BMJ 2015; 350:h1287.

59. Coventry P, Lovell K, Dickens C, Bower P, ChewGraham C, McElvenny D, Hann M, Cherrington A, Garrett C, Gibbons CJ, Baguley C, Roughley K, Adeyemi I, Reeves D, Waheed W, Gask L. Integrated primary care for patients with mental and physical multimorbidity: Cluster randomised controlled trial of collaborative care for patients with depression comorbid with diabetes or cardiovascular disease. $B M J$ 2015; 350:h638.

60. Fuller JD, Perkins D, Parker S, Holdsworth L, Kelly B, Roberts R, Martinez L, Fragar L. Effectiveness of service linkages in primary mental health care: a narrative review part 1. BMC Health Serv Res 2011; 11(1):72. 
61. Foy R, Hempel S, Rubenstein L, Suttorp M, Seelig M, Shanman R, Shekelle PG. Meta-analysis: effect of interactive communication between collaborating primary care physicians and specialists. Ann Intern Med. 2010; 152: 247-258.

62. Fuller JD, Perkins D, Parker S, Holdsworth L, Kelly B, Roberts R, Martinez L, Fragar L. Building effective service linkages in primary mental health care: a narrative review part 2. BMC Health Serv Res 2011; 11(1):66.

63. Matuda CG, Pinto NR da S, Martins CL, Frazão P. Colaboração interprofissional na Estratégia Saúde da Família: implicações para a produção do cuidado e a gestão do trabalho. Cien Saude Colet 2015; 20(8):2511-2521.

64. Coupe N, Anderson E, Gask L, Sykes P, Richards DA, Chew-Graham C. Facilitating professional liaison in collaborative care for depression in UK primary care; a qualitative study utilising normalisation process theory. BMC Family Practice 2014; 15(1):78. 
